\title{
Building a Classification Cascade for Visual Identification from One Example
}

\author{
Andras Ferencz \\ Computer Science, U.C. Berkeley \\ ferencz@cs.berkeley.edu
}

\author{
Erik G. Learned-Miller \\ Computer Science, UMass Amherst \\ elm@cs.umass.edu
}

\author{
Jitendra Malik \\ Computer Science, U.C. Berkeley \\ malik@cs.berkeley.edu
}

\begin{abstract}
Object identification (OID) is specialized recognition where the category is known (e.g. cars) and the algorithm recognizes an object's exact identity (e.g. Bob's BMW). Two special challenges characterize OID. (1) Inter-class variation is often small (many cars look alike) and may be dwarfed by illumination or pose changes. (2) There may be many classes but few or just one positive "training" examples per class. Due to (1), a solution must locate possibly subtle objectspecific salient features (a door handle) while avoiding distracting ones (a specular highlight). However, (2) rules out direct techniques of feature selection. We describe an online algorithm that takes one model image from a known category and builds an efficient "same" vs. "different" classification cascade by predicting the most discriminative feature set for that object. Our method not only estimates the saliency and scoring function for each candidate feature, but also models the dependency between features, building an ordered feature sequence unique to a specific model image, maximizing cumulative information content. Learned stopping thresholds make the classifier very efficient. To make this possible, category-specific characteristics are learned automatically in an off-line training procedure from labeled image pairs of the category, without prior knowledge about the category. Our method, using the same algorithm for both cars and faces, outperforms a wide variety of other methods.
\end{abstract}

\section{Introduction}

Object identification is specialized object recognition where the category is known (e.g. faces or cars) and one must recognize the exact identity of objects. The classes to be distinguished are not categories, e.g. cars versus non-cars (the problem of Object Categorization), but rather specific objects, like Bob's BMW or Jen's Ford. The hierarchical nature of categories suggests a continuum between these two problems: vehicles to cars to sedans to Phil's sedan. In this paper, we focus on the identification end of this continuum, where the Object Identification (OID) problem poses differ-

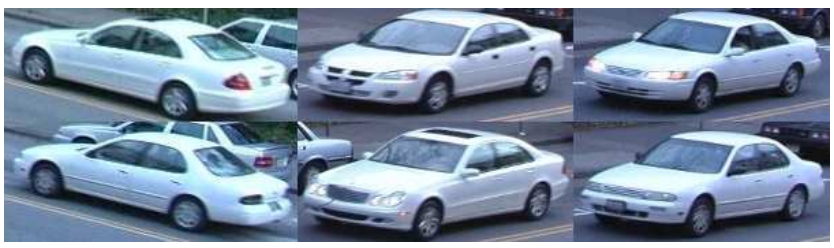

Figure 1: An Identification Problem: Which cars match? The two cars on the left were photographed from camera 1. Which of the four images on the right, taken by camera 2 , match the cars on the left?

ent challenges than its coarser cousin, Object Categorization (OC). Specifically, in OID problems (1) the inter-class variation is often small (many cars look alike), and this variation is often dwarfed by illumination or pose changes (see Fig. 1); and (2) there are many classes (each object is a separate class) but few (in our case just one) positive "training" examples per class (e.g. one image representing "Bob's BMW").

People are good at identifying individual objects from familiar categories after seeing them only once. Consider faces. We zero in on discriminative features for a person such as a prominent mole or unusually thick eyebrows, yet are not distracted by equally unusual but non-repeatable features such as a messy strand of hair or illumination artifacts. Domain specific expertise makes this possible: having seen many faces one learns that a messy strand of hair is not often a reliable feature. Human vision researchers report that acquisition of this expertise is accompanied by significant behavioral and physiological changes. Diamond et al. [3] showed that dog experts perform dog identification differently than non experts; Tarr et al. [12] argued that the brain's fusiform face area does visual processing of categories for which expertise has been gained.

The processes that occur during Object Categorization (OC) and Object Identification (OID) can be formally characterized. In functional notation, the stages for $\mathrm{OC}$ are

1. (Off-line) trainer $\mathcal{T}_{\text {cat }}$ : class training images $\mapsto \mathcal{C}_{\text {cat }}$,

2. (On-line) classifier $\mathcal{C}_{\text {cat }}$ : test image $\mapsto$ class label. 
There is nothing novel here, just the standard paradigm of statistical learning. It relies implicitly on having enough examples of each class to learn discriminative features.

For OID, we assume off-line access to plenty of examples of the category (cars, dogs, faces). We then must develop an on-line classifier for a future image of Bob's BMW, given only one example of it. We decompose the on-line process into two stages: (a) producing an "identifier", a classifier specialized to reidentify a specific object based on a single example of it, and (b) running the "identifier" on the incoming data stream. These on-line stages are preceded by the off-line process of learning category specific characteristics, resulting in an "identifier generator". Thus, the three stages for OID are

1. (Off-line) trainer $\mathcal{T}_{i d}$ : category training images $\mapsto \mathcal{H}_{i d}$,

2. (On-line) identifier generator $\mathcal{H}_{i d}$ : object image $\mapsto \mathcal{C}_{i d}$,

3. (On-line) classifier $\mathcal{C}_{i d}$ : test image $\mapsto$ s same, different $\}$.

We stress that step 1 learns category specific characteristics, while step 2 creates an object specific classifier. Now we address details.

First we need to pick a family of classifiers $\mathcal{C}_{i d}$. Motivated by the success of patch (a.k.a. part or fragment) based representations $([13,14])$ for OC, we use them for OID as well. Specifically, we develop an OID system whose generated classifier $\mathcal{C}_{i d}$ (step 3) is a patch-based classification cascade similar to that of Vidal-Naquet et al. [13], where evidence from features is accumulated incrementally until a "same" or "different" decision can be made. The tricky part is to give $\mathcal{H}_{i d}$ the ability to pick out object specific discriminative features (e.g. a prominent door handle in one car, a roof rack in another). But how can we know that a patch containing a prominent door handle is discriminative, based on a single image, when we have never seen a door handle exactly like it before?

The core of our approach is to use hyper-features, which are generic position and appearance characteristics of a patch. Examples include location of a patch, edge contrast in the patch and the dominant oriented energy in the patch. We might, in the process of becoming a car identification expert, expect to learn that patches about half-way up with strong edge contrast and a dominant horizontal orientation are particularly informative. When given the specific example of Bob's BMW, the identifier generator $\mathcal{H}_{i d}$ could produce an object-specific cascade with the first test based on the patch containing the door handle. Whereas for Jen's Ford, the same set of hyper-features will result in a different ordering of salient patches, resulting in a different classification cascade with the first test using a patch containing the roof rack (see Fig. 3).

More precisely, to instantiate $\mathcal{C}_{i d}$ (step 2), the function $\mathcal{H}_{i d}$ is given a single image of the object (e.g. Bob's BMW) and produces a sequence of patches ordered from most infor- mative to least, that maximizes the cumulative information content. This sequence is object-specific, and may emphasize different parts of each object.

The off-line training $\mathcal{T}_{i d}$ (step 1), given a set of image pairs from the category, each pair labeled "same" or "different," produces a class-specific $\mathcal{H}_{i d}$ by learning (a) a saliency and scoring model for image patches as a function of patch characteristics like position and appearance (hyperfeatures), (b) a dependency model between image patches based on similarity of their hyper-features, and (c) a set of thresholds for the cascade. The specific hyper-features used are themselves automatically selected during this training step from a large pool of candidate patch characteristics.

In contrast to some other "one-shot" learning algorithms $[11,4]$, where off-line training involves finding priors for a fixed model, our $\mathcal{T}_{i d}$ actually learns how to identify an arbitrary number of good features for the given category. Thus our final classifier $\mathcal{C}_{i d}$, while always a cascade of image patches taken from the model object, will have a different set of patches (in size, location, and count) for each object. To score a patch from a model image and its correspondent in a test image, our technique uses generalized linear models (GLMs) to estimate a generative model for the dissimilarity between patch pairs. "Same" and "different" distributions based on the hyper-features of the patch are estimated. These distributions are used both to estimate the saliency of a patch (by computing the expected mutual information between the dissimilarity and decision variables) and to score a patch pair by comparing the likelihood under the same and different distributions. By estimating bivariate "same" and "different" distributions for neighboring patches, we model the dependency relationships, allowing us to compute a sequence of patches with high joint information content.

Section 2 summarizes our previously published work on using hyper-features for visual identification [5]. That work had a serious limitation: it assumed that the patches were independent. This assumption is clearly false, especially for nearby and overlapping patches. To make that system work, we allowed only a single patch size and added a simple penalty term for a patch that was not a local maximum. Here we allow the system to pick patches of varying sizes, forcing us to model the patch dependencies. This model and its estimation from the training data is described in Section 3. With this dependency model, we build the cascade in Section 4 by finding stopping thresholds for making "same" or "different" decisions. Section 5 details our extensive experiments on car and face data sets.

\section{Learning Hyper-Features}

We begin by outlining the basic components of our system, some of which were previously detailed in [5]. As the main focus of this paper is modeling patch dependency (Section 3) and building the classification cascade (Section 4), we only 
summarize these components and ask the reader to refer to [6] for additional details and motivations for our choices. We describe the training $\left(\mathcal{T}_{i d}\right)$, identifier generating $\left(\mathcal{H}_{i d}\right)$, and classification $\left(\mathcal{C}_{i d}\right)$ functions in reverse order, starting with the final form of the object-specific classifier. In the following, we assume that all images are known to contain objects of the given category (e.g. cars or faces) and have been brought into rough correspondence (see Section 5 for details).

\subsection{Classifier $\mathcal{C}_{i d}$}

The classifier $\mathcal{C}_{i d}$ decides if a test (a.k.a. right) image $I^{R}$ is the same $(C=1)$ or different $(C=0)$ than the model (a.k.a. left) image $I^{L}$ it was trained for.

Patches. Our classifier consists of a sequence of image patches from the probe image $I^{L}$ and denoted $F_{j}^{L}$ for $1 \leq j \leq m$. Unlike our previous algorithm from [5], these patches can have different sizes and resolutions (by using different levels of a Gaussian pyramid). Generally speaking, larger patches are sampled at lower resolutions, keeping the complexity of the patches approximately constant. The gray-scale (we currently don't use color information) pixels of the patch are encoded by applying a first derivative Gaussian odd-symmetric filter at four orientations (horizontal, vertical, and two diagonal), giving four signed numbers per pixel.

Matching. Each encoded patch $F_{j}^{L}$ is matched to an equally sized area in the test image $I^{R}$, by searching for the most similar patch $F_{j}^{R}$ within some small neighborhood around the expected location (according to the coarse alignment). The distance function that this search minimizes is one minus the normalized correlation $d_{j}=1-$ $\operatorname{Corr} \operatorname{Coef}\left(F_{j}^{L}, F_{j}^{R}\right)$ between the encoded patches. The appearance distance $d_{j}$ is used as evidence for deciding if $I^{L}$ and $I^{R}$ are the same $(C=1)$ or different $(C=0)$.

Likelihood Ratio Score. To convert $d_{j}$ to a score, $\mathcal{C}_{i d}$ stores probability distributions $P\left(D_{j} \mid C=1\right)$ and $P\left(D_{j} \mid C=0\right)$ for each patch and computes the log likelihood ratio. (Note: $d_{j}$ refers to the specific measured distance for a given model and test image, while $D_{j}$ denotes the random variable from which $d_{j}$ is a sample). After $m$ patches have been matched, assuming independence, we score the match between images $I^{L}$ and $I^{R}$ using the sum of log likelihood ratios of matched patches:

$$
R=\sum_{j=1}^{m} \log \frac{P\left(D_{j}=d_{j} \mid C=1\right)}{P\left(D_{j}=d_{j} \mid C=0\right)} .
$$

To compute this, we must evaluate $P\left(D_{j}=d_{j} \mid C=1\right)$ and $P\left(D_{j}=d_{j} \mid C=0\right)$. In our system, both of these will take the form of gamma distributions $\Gamma\left(d_{j} ; \theta_{j}^{C=1}\right)$ and $\Gamma\left(d_{j} ; \theta_{j}^{C=0}\right)$, where the parameters $\theta_{j}^{C=1}$ and $\theta_{j}^{C=0}$ are defined as part of the classifier $\mathcal{C}_{i d}$ for each patch and are set by $\mathcal{H}_{i d}$ based on hyper-features.

Making a Decision. In [5], $\mathcal{C}_{i d}$ matched a fixed number of patches $(m)$, computed the score $\mathrm{R}$ by Eq. 1, and compared it to a threshold $\lambda . R>\lambda$ meant that $I^{L}$ and $I^{R}$ are the same. Otherwise they are declared different. In Section 4 of this paper, we define a cascade from the sequence of patches by applying thresholds after each patch has been matched.

To summarize, the classifier $\mathcal{C}_{i d}$ is defined by a sequence of patches of varying sizes (denoted $F_{j}^{L}$ ) taken from the probe image $I^{L}$. Additionally, a pair of parameters $\Theta_{j}^{C=1}$ and $\Theta_{j}^{C=0}$ that define the distributions $P\left(D_{j} \mid C=1\right)$ and $P\left(D_{j} \mid C=0\right)$ are associated with each patch.

\subsection{Classifier Generator $\mathcal{H}_{i d}$}

The classifier generator $\mathcal{H}_{i d}$ must take in a single model image $I^{L}$ of a new object from the given category and produce a sequence of patches $F_{1}^{L}, \ldots, F_{m}^{L}$ and their associated gamma distribution parameters, $\Theta_{1}^{C=1}, \ldots, \Theta_{m}^{C=1}$ and $\Theta_{1}^{C=0}, \ldots, \Theta_{m}^{C=0}$, for scoring based on the appearance distance measurement $d_{j}$ (which is measured when the patch $F_{j}^{L}$ is matched to a location in a test image $I^{R}$ ).

Estimating $\Theta_{j}^{C=1}$ and $\Theta_{j}^{C=0}$. Since being able to estimate a good $\Theta_{j}^{C=1}$ and $\Theta_{j}^{C=0}$ for any patch $j$ is also the key to picking good patches, we start with this step. Conceptually, we want $\Theta_{j}^{C}$ to be influenced by what patch $F_{j}^{L}$ looks like and where it is on the object (see the discussion of hyper-features in Section 1). First, we extract a predefined set of hyper-features from the patch such as [x_pos, $x_{-}$pos $^{2}$, size, resolution, contrast ${ }^{3}$, vertical_energy, ...]. Let $\mathbf{Z}_{\mathbf{j}}=\left[Z_{1}, \ldots, Z_{l}\right]^{T}$ be a vector of these hyper-features for patch $j$, and let $\Theta_{j}^{C}$ be parameterized as $\Theta=\left\{\mu_{j}, \gamma_{j}\right\}$. Now we define a generalized linear model (GLM) [10], which links these hyper-features $\mathbf{Z}$ to the gamma distribution $(\Gamma())$ model for $P\left(D_{j} \mid C=1\right)$ and $P\left(D_{j} \mid C=0\right)$ :

$$
P\left(D_{j} \mid Z, C\right)=\Gamma\left(d_{j} ; \alpha_{\mathbf{C}}^{\mu} \cdot \mathbf{Z}_{\mathbf{j}}, \alpha_{\mathbf{C}}^{\gamma} \cdot \mathbf{Z}_{\mathbf{j}}\right),
$$

where the second and third arguments to $\Gamma()$ are mean $\mu$ and shape $\gamma$ parameters. Each $\alpha$ (there are four of these $\left.\alpha_{C=0}^{\mu}, \alpha_{C=0}^{\gamma}, \alpha_{C=1}^{\mu}, \alpha_{C=1}^{\gamma}\right)$ is a vector of parameters of length $l$ that weights each hyper-feature monomial $Z_{i}$. The key point to notice is that given a hyper-feature encoding (the definition of which patch characteristics to extract) and the linear weights $\alpha$, we can estimate the distributions $P\left(D_{j} \mid C=1\right)$ and $P\left(D_{j} \mid C=0\right)$ for any probe image patch $F_{j}^{L}$, based on its position and appearance.

Estimating Saliency. If we define the saliency of a patch as the amount of information about the decision $C$ likely to be gained if the patch were to be matched, then it is straightforward to estimate saliency given $P\left(D_{j} \mid C=1\right)$ and $P\left(D_{j} \mid C=0\right)$. Intuitively, if $P\left(D_{j} \mid C=1\right)$ and $P\left(D_{j} \mid C=0\right)$ are similar distributions, we don't expect much useful information from a value of $d_{j}$. On the other 
hand, if the distributions are very different, then $d_{j}$ can tell us a great deal about our decision. Formally, this can be measured as the mutual information between the decision variable $C$ and the random variable $D_{j}$. Formally, this can be measured as the mutual information between the decision variable $C$ and the random variable $D_{j}$ (we assume equal priors on $\mathrm{C}, P(C=0)=P(C=1)=0.5)$ :

$$
I\left(D_{j} ; C\right)=H\left(D_{j}\right)-H\left(D_{j} \mid C\right) .
$$

Here $H()$ is Shannon entropy. The key fact to notice is that this measure can be computed just from the estimated distribution of $D_{j}$ (which, in turn, were estimated from the position and appearance of the model patch $F_{j}^{L}$ ) before the patch has been matched.

Finding Good Patches. The above mutual information formula allows us to estimate the saliency of any patch. Thus defining a sequence of patches to examine in order, from among all candidate patches, is straightforward: for each candidate patch estimate the distributions $P\left(D_{j} \mid C\right)$ from the hyper-features; compute the mutual information $I\left(D_{j} ; C\right)$; sort the patches by $I\left(D_{j} ; C\right)$; and take the top $m$ patches. The problem with this procedure is that the patches are not independent: once we have matched a patch $F_{j}^{L}$, the amount of additional information we are expected to derive from matching a patch $F_{i}^{L}$ that overlaps $F_{j}^{L}$ is less then $I\left(D_{i} ; C\right)$ would suggest. We discuss a solution to this problem in Section 3.

However, assuming that this dependency problem can be solved, we have a complete algorithm for generating the classifier $\mathcal{C}_{i d}$ from a single image, given a definition of the hyper-features to extract (the patch statistics $\mathbf{Z}$ ) and the linear weights $\alpha$.

\subsection{Off-line Training $\mathcal{T}_{i d}$}

The task of the off-line training step $\mathcal{T}_{i d}$ is to define the hyper-feature encoding $\mathbf{Z}$ and to learn the weights $\alpha$ that link this encoding to the distributions $P\left(D_{j} \mid C=1\right)$ and $P\left(D_{j} \mid C=0\right)$. This step is given a large collection of image pairs from the category, where each left-right image pair is labeled as "same" or "different". A large number of patches $F_{j}^{L}$ are sampled from the left images and matched to the right images (by finding the best matching $F_{j}^{R}$ ) in the same manner as during classification $\mathcal{C}_{i d}$ (see Matching in Section 2.1), and the appearance distance $d_{j}$ is recorded. For each patch, a large set of candidate hyper-features are also extracted from the position and appearance of the left patch $F_{j}^{L}$. This data gives rise to 2 generalized linear regression problems: one for the "same" $(C=1)$ set and one for the "different" $(C=0)$ set. Our solution involves (1) a feature selection step which finds a hyper-feature encoding $(\mathbf{Z})$ by choosing a small subset from the candidate set of hyperfeatures, and (2) a maximum likelihood estimation step to fit $\alpha_{C=1}^{\mu}, \alpha_{C=1}^{\gamma}$ and $\alpha_{C=0}^{\mu}, \alpha_{C=0}^{\gamma}$.

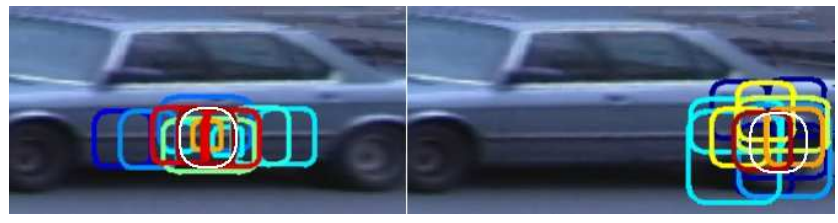

Figure 2: Patch Correlations. On each image, the patches most correlated with the white-circled patch are shown. Notice that in the left image, where the patch sits in an area with a highly visible horizontal structure, the most correlated patches all lie along the horizontal features. Contrast this with the right image, showing correlation of patches with a patch sitting on a wheel, where the most correlated patches are those that strictly overlap the whitecircled patch.

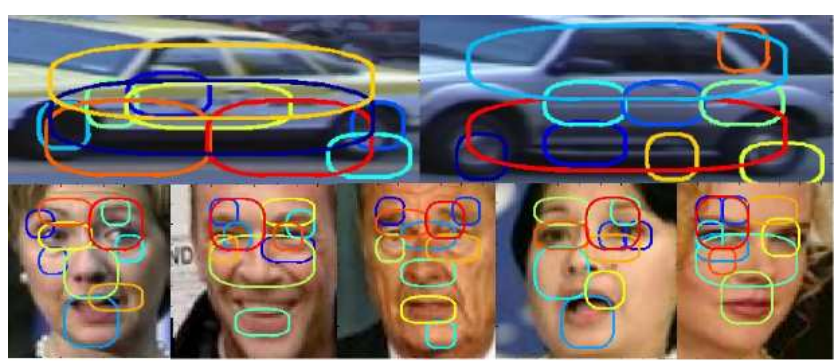

Figure 3: The Ten Most Informative Patches. The ten rectangles on each object show the top ten patches our algorithm selected for the classification cascade for that object. The face model seems to prefer features around the eyes, while the car models tend to like wheels. Notice, however, that even within a category each cascade is unique, highlighting interesting appearance features for that object. The patches are color coded according to their order, from dark red (1) to dark blue (10).

\section{Modeling Pairwise Relationships Between Patches}

In Section 2, we described our model to score a probe image patch $F_{j}^{L}$ and its best match $F_{j}^{R}$ by modeling the distribution of their distance in appearance, $D_{j}$, conditioned on the match variable $C$. Furthermore, in Section 2.2, we described how to infer the saliency of the patch $F_{j}^{L}$ for matching based on these distributions. As we noted in that section, this works for picking the first patch, but is not optimal for picking subsequent patches: once we have already matched and recorded the score of the first patch, the amount of information gained from a nearby patch is likely to be small, because their scores are likely to be correlated. Intuitively, the next chosen patch would ideally be a highly salient patch whose information about $C$ is as independent as possible from the first patch. Similarly, the third patch should consider both the first and the second patches.

Let $F_{(k)}^{L}$ represent the $k$ th patch picked for the cascade and let $F_{(1 \ldots n)}^{L}$ denote the first $n$ of these patches. As- 
sume we have already picked patches $F_{(1 \ldots n)}^{L}$ and we wish to choose the next one, $F_{(n+1)}^{L}$, from the remaining set of $F_{j}^{L}$ 's. We would like to pick the one that maximizes the information gain or the conditional mutual information:

$I\left(D_{(n+1)} ; C \mid D_{(1 \ldots n)}\right)=I\left(D_{(1 \ldots n+1)} ; C\right)-I\left(D_{(1 \ldots n)} ; C\right)$.

This quantity is difficult to estimate, due to the need to model the joint distribution of all $D_{(1 \ldots n)}$ patches. However, note that the information gain of a new feature is upper bounded by the information gain of that feature relative to any single feature that has already been chosen. That is,

$$
I\left(D_{(n+1)} ; C \mid D_{(1 \ldots n)}\right) \leq \min _{1 \leq i \leq n} I\left(D_{(n+1)} ; C \mid D_{(i)}\right) .
$$

Thus, rather than maximizing the full information gain, we select the new feature that maximizes this upper bound on the amount of "new" information:

$$
\arg \max _{j} \min _{i} I\left(D_{j} ; C \mid D_{(i)}\right),
$$

where $i$ varies over the already chosen patches, and $j$ varies over the remaining patches. This formulation (Eq. 4) follows that of Vidal-Naquet et al. [13].

\subsection{Dependency Model}

To compute (4), we need to estimate conditional mutual informations of the form

$$
I\left(D_{j} ; C \mid D_{(i)}\right)=I\left(D_{j}, D_{(i)} ; C\right)-I\left(D_{(i)} ; C\right) .
$$

In Section 2.2, we showed that we can determine the second term, $I\left(D_{(i)} ; C\right)$, from the estimated gamma distributions for $P\left(D_{(i)} \mid C=1\right)$ and $P\left(D_{(i)} \mid C=0\right)$. Similarly, to calculate $I\left(D_{j}, D_{(i)} ; C\right)$, we need an estimate of the bivariate distributions for $P\left(D_{(i)}, D_{j} \mid C=1\right)$ and $P\left(D_{(i)}, D_{j} \mid C=0\right)$. If the $D_{(i)}$ and $D_{j}$ are independent conditioned on $C$, then these are straightforward to compute from the known marginal distribution parameters for $D_{(i)}$ and $D_{j}$. To model the dependent case, we employ Kibble's bivariate gamma distribution [8], which has four parameters: $K\left(\mu_{1}, \mu_{2}, \gamma, \rho\right), 0<\rho<1 . \mu_{1}$ and $\mu_{2}$ are mean parameters for the marginals, and $\gamma$ is a dispersion parameter for both marginals (the formulation requires these to be equal). $\rho$ is the correlation between $D_{(i)}$ and $D_{j}$, and varies from 0 , indicating full independence of the marginals, to 1 , in which the marginals are completely correlated.

To make this formulation work, the marginal distribution parameters must be constrained to be equal $\left(\mu_{j}^{C=1}=\mu_{(i)}^{C=1}\right.$, as well as $\left.\gamma_{j}^{C=1}=\gamma_{(i)}^{C=1}\right)^{1}$. Therefore, for the computation of the conditional mutual information of $D_{j}$ conditioned on

\footnotetext{
${ }^{1}$ More precisely the $\gamma$ 's must be equal (this is a requirement of Kibble's formulation), while the $\mu$ 's must satisfy $\frac{\mu_{j}^{C=1}}{\mu_{(i)}^{C=1}}=\frac{\mu_{j}^{C=0}}{\mu_{(i)}^{C=0}}$
}

$D_{(i)}$, we force the marginal distribution of the already chosen patch $\left(D_{(i)}\right)$ to be equal to the marginal distribution of the patch currently being considered $\left(D_{j}\right)$. Given that our method for comparing all patches is the same, namely normalized correlation, this usually means a very minor perturbation to the estimated distribution of $D_{(i)}$ when the two patches are strongly correlated. On the other hand, when the marginals are originally fairly different, the two patches tend to be uncorrelated. In this case, the exact shapes of $D_{(i)}$ 's distributions are less relevant to the computation of Eq. (4). Since we are always setting the first two parameters of Kibble's distribution to be the same, we will henceforth write it with three parameters (e.g. $K\left(\mu_{j}^{C=0}, \gamma_{j}^{C=0}, \rho\right)$ ).

\subsection{Predicting Patch Correlations from Hyper-Feature Differences}

Given the above formulation, we have reduced the problem of finding the next best patch, $F_{(n+1)}^{L}$, to the problem of estimating the correlation parameter $\rho$ of Kibble's bivariate gamma distribution for any pair of patches $F_{(i)}^{L}$ (one of the $n$ patches already selected) and $F_{j}^{L}$ (a candidate for $F_{(n+1)}^{L}$ ). The intuition is that patches that are nearby and overlapping or that lie on the same underlying image features (for example the horizontal line on the side of the car in Figure 2) are likely to be highly correlated, whereas two patches that are of different sizes and far away from one another are likely to be less so.

We model $\rho$, the last parameter of $K\left(\mu_{j}^{C=1}, \gamma_{j}^{C=1}, \rho\right)$ and $K\left(\mu_{j}^{C=0}, \gamma_{j}^{C=0}, \rho\right)$, similarly to our GLM estimate of its other parameters (see Section 2.2): we let $\rho$ be a linear function of the difference of various hyper-features of the two patches, $F_{(i)}^{L}$ and $F_{j}^{L}$. Clear candidates for these covariates are the difference in position and size of the two patches, as well as some image-based features such as the difference in the amount of contrast within each patch. To ensure $0<\rho<1$, we use a sigmoid link function $\rho=(1-\exp (\beta \cdot \mathbf{Y}))^{-1}$, where $\mathbf{Y}$ is our vector of hyperfeature differences and $\beta$ is the GLM parameter vector.

Given a data set of patch pairs $F_{(i)}^{L}$ and $F_{j}^{L}$ and associated distances $d_{(i)}$ and $d_{j}$ (found by matching the "left" patches to a "right" image of the same or of a different object), we estimate the linear coefficients $\beta$. This is done by maximizing the likelihood of $K\left(\mu_{j}^{C=1}, \gamma_{j}^{C=1}, \rho\right)$ using data taken from image pairs that are known to be the "same" $\left(\mu_{j}^{C=1}\right.$ and $\gamma_{j}^{C=1}$ are estimated from $F_{j}^{L}$ by the method of Section 2.3 and are fixed for this optimization). and $K\left(\mu_{j}^{C=0}, \gamma_{j}^{C=0}, \rho\right)$ using data taken from "different" image pairs. Also similarly to Section 2.3, we choose the encoding of $\mathbf{Y}$ automatically, by the method of forward feature selection [7] over candidate hyper-feature difference variables. As anticipated, the top ranked variables encoded differences in position, size, contrast, and orientation energy. Our final model 
uses the top 10 variables.

\section{Building the Cascade}

Now that we have a model for patch dependence, we can create a sequence of patches $F_{j}^{L}$ (see Section 2.2) that, when matched, collectively capture the maximum amount of information about the decision $C$ (same or different?). The sequence is ordered so that the first patch is the most informative, the second slightly less so and so on. The final step of creating a cascade is to define early stopping thresholds on the log likelihood ratio sum $R$ that can be applied after each patch in the sequence has been matched and its score added to $R$ (see Section 2.1).

We assume that we are given a global threshold $\lambda$ (see Section 2.1) that defines a global choice between selectivity and sensitivity. What remains is the definition of thresholds at each step, $\lambda_{(k)}^{C=1}$ and $\lambda_{(k)}^{C=0}$, which allow the system to accept (declare "same") if $R>\lambda_{(k)}^{C=1}$ or reject (declare "different") if $R \leq \lambda_{(k)}^{C=1}$, otherwise continue by matching patch $k+1$. To learn these thresholds, we run $\mathcal{H}_{i d}$ on the left images and the resulting classifier $\mathcal{C}_{i d}$ on the right images of our training data set. This will produce a performance curve for each choice of $k$, the number of patches included in the classification score, including $k=m$, the sum for which $\lambda$ is defined. Our goal for the cascade is for it to make decisions as early as possible (tight thresholds) but, on the training set, never make a mistake on any pair which was correctly classified using all $m$ patches and the threshold $\lambda$. These two constraints exactly define the thresholds $\lambda_{(k)}^{C=1}$ and $\lambda_{(k)}^{C=0}$.

\section{Results and Conclusion}

The goal of this work was to create an identification system that could be applied to different categories, where the algorithm would automatically learn (based on off-line training examples) how to select category-specific salient features from a new image. In this section, we demonstrate that after category training, our algorithm is in fact able take a single image of a novel object and solely based on it create a highly effective "same" vs. "different" classification cascade of image patches. Specifically, we wish to show that for visual identification each of the following leads to an improvement in performance in terms of accuracy and/or computational efficiency:

1. breaking the object up into patches (a.k.a parts, fragments), matching each one separately and combining the results,

2. differentiating patches by estimating a scoring and saliency function for each patch (based on its hyperfeatures),

3. modeling the dependency between patches to create a sequence of patches to be examined in order, and

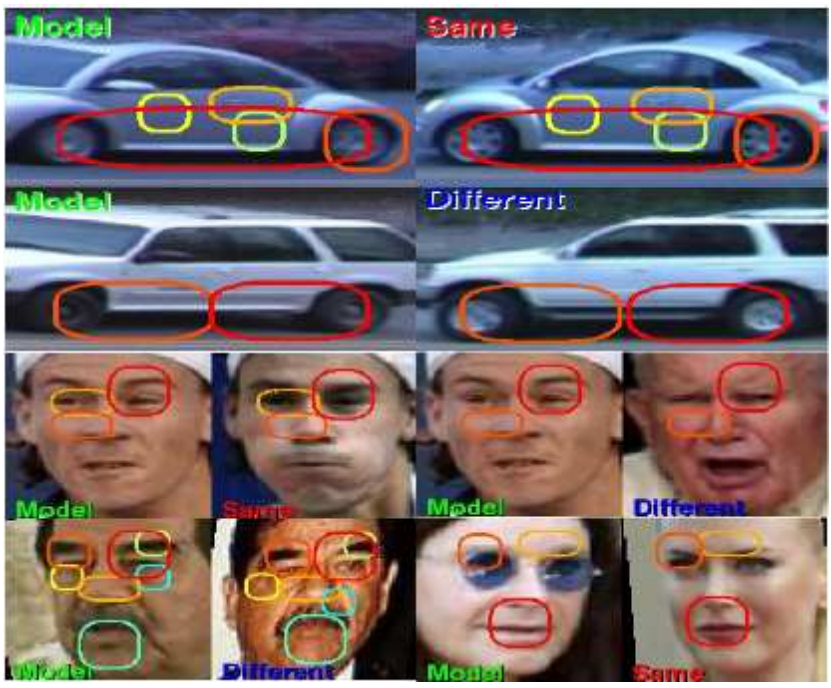

Figure 4: Example Model-Test Classifications. Each pair of images shows a model and a test image, which has been labeled as "same" or "different" by our algorithm. The patches that were used in the cascade for that test image are indicated for each pair, where the order is color coded from red to blue. The first 3 rows show correct classification results. For cars, false-negative errors (not shown) primarily occur with darker cars where the main source of features are the illumination artifacts that can vary greatly between the images, while false-positive errors tend to involve very similar vehicles (e.g. same make and model). Two typical face errors are shown in the last row: the large variations in pose, lighting, expression and image resolution make the face data set very difficult. Note: the model for the person wearing sunglasses in the last row is the only one whose first patch in the cascade is not on the eye.

4. applying early termination thresholds to the patch sequence to create the cascade.

We tested our algorithm on two different data sets: (1) cars from 2 cameras with significant pose differential, and (2) faces from news photographs. Examples from these two data sets are shown in Figure 3, with the top 10 patches of the classification cascade, and Figure 4, with model-test classification results. For each data set, a different automatic preprocessing step was applied to detect objects and approximately align them. After this, the same identification algorithm was applied to both sets. For lack of space, we detail our experiments on data set 1 , and only summarize the results for data set 2. Qualitatively, our results are consistent in showing that each of the above aspects of our system improves the performance, and that the overall system is both efficient and effective. In the last section, we compare our system to Lowe's technique [9], which does not use category-specific learning.

\subsection{Cars}

358 unique vehicles (179 training, 179 test) were extracted using a blob tracker from 1.5 hours of video from two cam- 


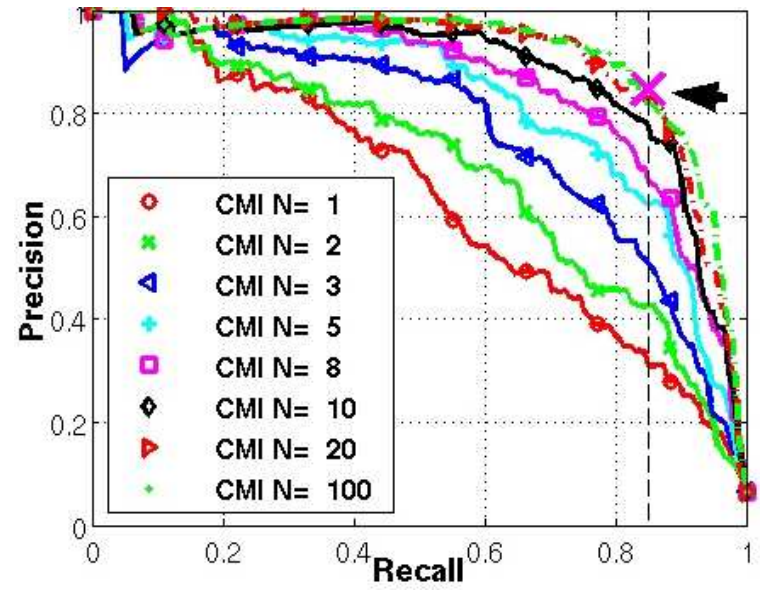

Figure 5: Precision vs. Recall Using Different Numbers of Patches. These are precision vs. recall curves for our full model. Each curve represents the performance tradeoff between precision and recall, when the system uses a fixed number of patches. The lowest curve uses only the single most informative patch, while the top curve uses up to 100 patches. The $85 \%$ recall rate, where the different models of Figure 6 are compared, is noted by a vertical black dashed line. A magenta $\mathrm{X}$, at recall $=84.9$ and precision $=$ 84.8, marks the performance of the cascade model.

eras located one block apart. The pose of the cameras relative to the road (see Figure 1) was known from static camera calibration, and alignment included warping the sides of the vehicles to be approximately parallel to the image plane. Within training and testing sets, about 2685 pairs (true to false ratio of 1:15) of mismatched cars were formed from non-corresponding images, one from each camera. These included only those car pairs that were superficially similar in intensity and size. Using the best whole image comparison method we could find (normalized correlation on blurred filter outputs) on this set produces $14 \%$ false positives at a $15 \%$ miss rate. This data set is available from wWw. cs. berkeley. edu/ ferencz/vid.

Figure 6 compares several versions of our model by plotting the false-positive rate (y-axis) with a fixed miss rate of $15 \%$ ( $85 \%$ recall), for a fixed budget of patches (x-axis). The $85 \%$ recall point was selected based on Figure 5 , by picking the equal error point given the 1 to 15 true-to-false ratio. The Random Order curve uses our hyper-feature model for scoring, but chooses the patches randomly. By comparing this curve to its neighbors, notice the performance gain associated with differentiating patches based on hyper-features both for scoring (No Hyper-Features vs. Random Order) and for patch selection (Random Order vs. Mutual Information). Comparing Mutual Information vs. Conditional MI shows that modeling patch dependence is important for choosing a small number of patches (see range 5-20) that together have high information content (Section 3 ). Comparing Position Only (which only uses positional hyper-features) vs.

\begin{tabular}{|c|cccc|}
\hline Recall Rate & $60 \%$ & $70 \%$ & $80 \%$ & $90 \%$ \\
\hline PCA + MahCosine & $82 \%$ & $73 \%$ & $62 \%$ & $59 \%$ \\
Filter + NormCor & $83 \%$ & $73 \%$ & $67 \%$ & $57 \%$ \\
No Hyper-Features & $86 \%$ & $73 \%$ & $68 \%$ & $62 \%$ \\
Random 10 Patches & $79 \%$ & $71 \%$ & $64 \%$ & $60 \%$ \\
Top 1 CMI Patch & $86 \%$ & $76 \%$ & $69 \%$ & $63 \%$ \\
Top 50 CMI Patches & $92 \%$ & $84 \%$ & $75 \%$ & $67 \%$ \\
CMI Cascade & $\mathbf{9 2 \%}$ & $\mathbf{8 4 \%}$ & $\mathbf{7 6 \%}$ & $\mathbf{6 6 \%}$ \\
\hline
\end{tabular}

Table 1: Precision vs. Recall for Faces.

Each column denotes the precision associated with a given recall rate along the P-R curve. PCA + MahCosine and Filter + NormCor are whole face comparison techniques. $P C A+$ MahCosine is the best curve produced by [2], which implements PCA and LDA algorithms with face-specific preprocessing. Filter + NormCor uses the same representation and comparison method as our patches, but applied to the whole face. The last 4 all use our patch based system with hyper-features. The last 3 uses conditional mutual information based patch selection, where the number of patches allowed is set to 1,50 , and variable (cascade), respectively.

Conditional MI (which uses both positional and appearance hyper-features) shows that patch appearance characteristics are significant for both scoring and saliency estimation. Finally, the cascade performs (1.02\% error, with mean of 4.3 patches used) as well as the full model and better than any of the others, even when these are given an unlimited computation budget.

Figure 5 shows another way to look at the performance of our full model given a fixed patch (computation) budget (the Conditional MI curve of Figure 6 represents the intersection of these curves with the $85 \%$ recall line). The cascade performance is also plotted here (follow the black arrow).

\subsection{Faces}

We used a subset of the "Faces in the News" data set described in [1], where the faces have been automatically detected from news photographs and registered by their algorithm. Our training and test sets each used 103 different people, with two images per person. This is an extremely difficult data set for any identification algorithm, as these face images were collected in a completely uncontrolled manner (news photographs). Table 1 summarizes our results for running the same algorithm as above on this set. Note the same pattern as above: the patch based system generally outperforms whole object systems (here we compare against state of the art PCA and LDA algorithms with face specific preprocessing using CSU's implementation [2]); estimating a scoring and saliency function through hyper-features greatly improves the performance of the patch based system; the cascades, using less than 6 patches on average, performs as well as always using the best 50 patches (performance actually declines above 50 patches). 


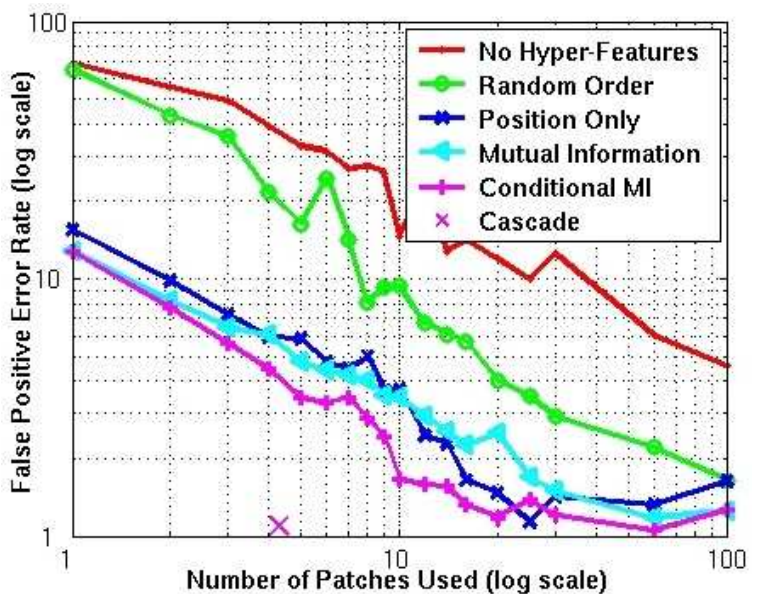

Figure 6: Comparing Performance of Different Models. The curves plot the performance of various models, as measured by the false-positive rate (fraction of different pairs labeled incorrectly as same), at a fixed recall rate of $85 \%$. The $y$-axis shows the log error rate, while the $x$-axis plots the log number of patches the models were allowed to use (up to a max of 100). As the number of patches increases, the performance improves until a point, after which it levels off and, for the models that order patches according to information gain, even decreases (when non-informative patches begin to pollute the score). The (red) model that does not use hyperfeatures (i.e. uses the same distributions for all patches), performs very poorly compared to the hyper-feature versions, even when it is allowed to use 100 patches. The second curve from the top uses our hyper-feature model to score the patches, but random selection to pick the patch order. The position only model uses only positionbased hyper-features for selecting patch order (i.e. it computes a fixed patch order for all cars). The light blue model sorts patches by mutual information, without considering dependencies. The last curve shows our full model based on selecting patches according to their conditional mutual information, using both positional and image-based hyper-features. Finally, the magenta $X$ at 4.3 patches and $1.02 \%$ error shows the performance of the cascade model.

\subsection{Comparison to SIFT}

In the previous sections, we have shown that each step of our algorithm improves the classification performance. But how does our system compare with other leading methods? While an in-depth comparison is beyond the scope of this paper, we compared our results to David Lowe's technique applied to our car identification problem. We used a version of his algorithm described in [9] with no category-specific learning. At the $85 \%$ recall point, that algorithm produced a false-positive error rate of over $20 \%$ on our data set (compared to our $1.02 \%$ ). Limiting the area and rotation of the matching SIFT features given the approximate alignment that is used in our algorithm did not improve these results significantly. In addition to the obvious lack of categoryspecific training, the poor performance of Lowe's algorithm on this data may be due to the design of the SIFT features. Specifically, we found that due to the nature of the problem, where distinct objects can look very similar except for a few subtle differences, features that were developed to be robust to small differences (such as SIFT) do not perform well.

\section{Acknowledgments}

This work was supported by the DARPA CZTS project. We thank Ryan White for providing the face data set, Hao Zhang for an implementation of LARS and Vidit Jain for implementing the comparison with SIFT.

\section{References}

[1] T. Berg, A. Berg, J. Edwards, M. Maire, R. White, Y. Teh, E. Learned-Miller, and D. Forsyth. Names and faces in the news. CVPR, 2004.

[2] D. Bolme, R. Beveridge, M. Teixeira, and B. Draper. The csu face identification evaluation system: Its purpose, features and structure. ICVS, 2003.

[3] R. Diamond and S. Carey. Why faces are and are not special: An effect of expertise. Journal of Experimental Psychology, Gen(115):107-117, 1986

[4] L. Fei-Fei, R. Fergus, and P. Perona. A Bayesian approach to unsupervised one-shot learning of object categories. In ICCV, 2003.

[5] A. Ferencz, E. Learned-Miller, and J. Malik. Learning hyperfeatures for visual identification. NIPS, 2004.

[6] A. Ferencz, E. Learned-Miller, and J. Malik. Learning to locate informative features for visual identification. Technical report, UC Berkeley, Electronics Research Laboratory, 2005.

[7] G. John, R. Kohavi, and K. Pfleger. Irrelevant features and the subset selection problem. In ICML, pages 121-129, 1994.

[8] W. F. Kibble. A two-variate gamma type distribution. Sankhya, 5:137-150, 1941.

[9] D. Lowe. Distinctive image features from scale-invariant keypoints. IJCV, 60(2):91-110, 2004.

[10] P. McCullagh and J. A. Nelder. Generalized Linear Models. Chapman and Hall, 1989.

[11] E. Miller, N. Matsakis, and P. Viola. Learning from one example through shared densities on transforms. In IEEE Computer Vision and Pattern Recognition, 2000.

[12] M. Tarr and I. Gauthier. FFA: A flexible fusiform area for subordinate-level visual processing automatized by expertise. Nature Neuroscience, 3(8):764-769, 2000.

[13] M. Vidal-Naquet and S. Ullman. Object recognition with informative features and linear classification. In ICCV, 2003.

[14] M. Weber, M. Welling, and P. Perona. Unsupervised learning of models for recognition. ECCV, 2000. 